\title{
COMPLETE LATTICES AND THE GENERALIZED CANTOR THEOREM
}

\author{
ROY O. DAVIES, ALLAN HAYES AND GEORGE ROUSSEAU
}

Abstract. Cantor's Theorem is generalized to a theorem on partially ordered sets.

We shall show that every monotone mapping of a complete lattice into itself has a point of left continuity and a point of right continuity. From this result we derive an extension of a theorem of Gleason and Dilworth [2] which in turn can be regarded as a generalization of the classical theorem of Cantor stating that the cardinal of a set is less than the cardinal of its power-set. As a corollary it follows that if $E$ and $F$ are partially ordered sets then the cardinal power $F^{E}$ is not a homomorphic image of $E$ unless $|F|=1$. This result answers a question of F. W. Lawvere which provided the stimulus for our investigation.

1. A continuity theorem for complete lattices. If $E$ and $F$ are partially ordered sets then a mapping $\phi: E \rightarrow F$ is said to be isotone if for all $x, y \in E$

$$
x \leqq y \text { implies } \phi(x) \leqq \phi(y) ;
$$

the point $a \in E$ is called a point of left continuity resp. right continuity for $\phi$ if

$$
\phi(a)=\bigvee_{x<a} \phi(x) \quad \operatorname{resp} . \phi(a)=\bigwedge_{x>a} \phi(x) .
$$

A mapping $\phi: E \rightarrow F$ is said to be antitone if for all $x, y \in E$

$$
x \leqq y \text { implies } \phi(x) \geqq \phi(y) ;
$$

the point $a \in E$ is called a point of left continuity resp. right continuity for $\phi$ if

$$
\phi(a)=\bigwedge_{x<a} \phi(x) \quad \operatorname{resp} . \phi(a)=\bigvee_{x>a} \phi(x) .
$$

(In the familiar case of monotone functions on the real line, these definitions of left and right continuity are equivalent to the usual ones.) If the inequalities in (1) and (2) are replaced by strict in-

Received by the editors January 13, 1969.

AMS 1968 subject classifications. Primary 0620; Secondary 0630.

Key words and phrases. Cantor's Theorem, left continuity, monotone mappings

Copyright (c) 1971, American Mathematical Society 
equalities then we obtain the notions of strictly isotone and strictly antitone mapping. A mapping is called monotone if it is isotone or antitone.

THEOREM 1. Every monotone mapping of a complete lattice into itself has a point of left continuity and a point of right continuity.

Proof. Let $C$ be a complete lattice and let $\phi: C \rightarrow C$ be a monotone mapping. We prove the existence of a point of left continuity; the existence of a point of right continuity may be established by a dual argument.

Suppose first that $\phi$ is isotone, and let $a=\mathrm{V}_{x<\phi(x)} \phi(x)$. Then

$$
a=\underset{x<\phi(x)}{\bigvee} \phi(x) \leqq \bigvee_{x<a} \phi(x) \leqq \phi(a)
$$

If $a<\phi(a)$ then $\phi(a) \leqq a$, a contradiction. Hence equality holds throughout in (3), and consequently $a$ is a point of left continuity for $\phi$.

Suppose now that $\phi$ is antitone. We may suppose that $\phi$ is strictly antitone; for otherwise there exist elements $a$ and $b$ such that $a<b$ and $\phi(a)=\phi(b)$, in which case $\phi(b)=\phi(a) \geqq \bigwedge_{x<b} \phi(x) \geqq \phi(b)$, so that $b$ is a point of left continuity for $\phi$. Since $\phi^{2}$ is isotone, there exists, by what has already been proved, an element $a$ such that

$$
\phi(\phi(a))=\bigwedge_{x>a} \phi(\phi(x)) \geqq \underset{y<\phi(a)}{\bigvee} \phi(y) \geqq \phi(\phi(a)) ;
$$

hence $\phi(a)$ is a point of left continuity for $\phi$.

CoROLlARY. If $f$ is an arbitrary mapping of a complete lattice into itself then there exist points $a$ and $b$ such that

$$
f(a) \leqq \bigvee_{x<a} f(x) \quad \text { and } \quad f(b) \leqq \bigvee_{x>b} f(x)
$$

Proof. Apply Theorem 1 to the monotone mappings

$$
\phi_{1}(x)=\bigvee_{u \leqq x} f(u) \text { and } \quad \phi_{2}(x)=\bigvee_{u \geq x} f(u)
$$

We observe that in the case of an isotone mapping $\phi$, the proof of Theorem 1 establishes the existence of a fixed point at which $\phi$ is left continuous. Thus we obtain the fixed point theorem of Tarski [4].

The Tarski fixed point theorem characterizes complete lattices (cf. Davis [1]), but Theorem 1 does not; for example, every monotone mapping of the incomplete lattice $1+\left(\omega^{*}+\omega\right) \cdot 2+1$ has a point 
of left continuity and a point of right continuity. Again, the fixed points of an isotone mapping form a complete lattice (cf. Tarski [4]), whereas the points of left continuity need not; for example, consider the function $\operatorname{sgn}(x)$ on the real interval $-1 \leqq x \leqq 1$.

2. An extension of a theorem of Gleason and Dilworth. A left ideal in a partially ordered set $E$ is a subset $L$ such that if $x \leqq y$ and $y \in L$ then $x \in L$; a right ideal is a subset $R$ such that if $x \geqq y$ and $y \in R$ then $x \in R$.

The set $\mathscr{L}(E)$ of left ideals and the set $R(E)$ of right ideals are easily seen to be complete lattices when partially ordered by inclusion; these lattices are in fact anti-isomorphic.

Henceforth we shall refer to isotone mappings as homomorphisms.

Gleason and Dilworth [2] prove a theorem which is easily seen to be equivalent to the following statement:

( $\lambda$ ) If $E$ is a partially ordered set, then there is no injective homomorphism from $\mathcal{L}(E)$ to $E$ and no surjective homomorphism from $E$ to $\mathfrak{L}(E)$.

From this result we may derive the corresponding statement for right ideals:

(o) If $E$ is a partially ordered set, then there is no injective homomorphism from $R(E)$ to $E$ and no surjective homomorphism from $E$ to $R(E)$.

Indeed, if there were an injective homomorphism $\phi: R(E) \rightarrow E$ then there would be an injective homomorphism $\psi: \&(E) \rightarrow E$, defined by

$$
\psi(L)=\phi(C L \phi(C L)),
$$

where $C X=E-X$ and $L x=\{u \in E: u \leqq x\}$; similarly, if there were a surjective homomorphism $\phi: E \rightarrow R(E)$ then there would be a surjective homomorphism $\psi: E \rightarrow \mathfrak{L}(E)$, defined by

$$
\psi(x)=\mathbf{C} \mathbf{U} \phi[\mathbf{C \phi}(x)],
$$

where $\phi[X]=\{\phi(x): x \in X\}$ and $\mathbf{U} \mathfrak{X}=\cup_{X \in X} X$. (The mapping $\psi$ is surjective because, given $L$, we can find $x$ such that $\psi(x)=L$ by choosing $z$ so that $\phi(z)=C L$ and $x$ so that $\phi(x)=C L z$.) It is interesting to note that it does not seem possible to derive $(\lambda)$ from $(\rho)$ in this manner.

Using the result of $\$ 1$ we now prove a theorem which includes both $(\lambda)$ and $(\rho)$, and which seems to be more general.

If $E$ and $F$ are partially ordered sets then a mapping $\phi: E \rightarrow F$. 
will be called a weak monomorphism if the following condition holds for all $x, y \in E$ : if $x \leqq y$ and $\phi(x) \geqq \phi(y)$ then $x=y$.

It is convenient to admit mappings which are not necessarily single-valued, and in this case $\phi(x)$ denotes ambiguously any image of $x$ under $\phi$. The next theorem will be stated with this understanding. If we assume the axiom of choice it is not necessary to consider manyvalued mappings; we discuss this question after the proof of Theorem 2 .

The class of weak monomorphisms contains all injective homomorphisms and the inverses of surjective homomorphisms. It is therefore clear that the following result includes $(\lambda)$ and $(\rho)$ :

THEOREM 2. If $E$ is a partially ordered set, then there is no weak monomorphism from $\mathfrak{L}(E)$ to $E$ or from $R(E)$ to $E$.

Proof. Given any mapping $\phi: \mathfrak{L}(E) \rightarrow E$, define a mapping $f$ of the complete lattice $\mathscr{L}(E)$ into itself by letting $f(L)$ be the set of all $x \in E$ such that $x \leqq \phi(L)$ for some value of $\phi(L)$. By the Corollary to Theorem 1 , there exists $L$ such that

$$
f(L) \subseteq \bigcup_{M \subset L} f(M)
$$

Choosing any value of $\phi(L)$ we have $\phi(L) \in f(L)$; hence there exists $M \subset L$ such that $\phi(L) \in f(M)$; it follows that for some value of $\phi(M)$ we have $\phi(L) \leqq \phi(M)$; hence we have $M \subset L$ and $\phi(M) \geqq \phi(L)$, and thus $\phi$ is not a weak monomorphism. By a similar argument there can be no weak monomorphism from $R(E)$ to $E$.

If we assume the axiom of choice then Theorem 2 can be deduced from the special case referring to single-valued mappings; indeed given a many-valued weak monomorphism $\phi: \mathcal{L}(E) \rightarrow E$ we can obtain a single-valued weak monomorphism $\phi_{0}: \mathcal{L}(E) \rightarrow E$ by choosing as the value of $\phi_{0}(L)$ one particular value of $\phi(L)$. Hence in particular $(\rho)$, as well $(\lambda)$, can be deduced from this restricted form of Theorem 2 if we have recourse to the axiom of choice.

We note the following consequence of Theorem 2 which does not seem to be derivable from the theorem of Gleason and Dilworth, or by simple considerations of cardinality:

(*) There is no function $\phi$ from the power-set of a set $E$ into $E$ such that $\phi$ is one-one on every chain.

That is, there is no function $\phi: P(E) \rightarrow E$ such that for all $X$, $Y \in P(E)$

$$
X \subset Y \text { implies } \phi(X) \neq \phi(Y) \text {. }
$$


This proposition can be derived from the following theorem of Sierpiński [3]:

For every set $E$ there exists a chain $\Gamma$ of subsets of $E$ with cardinality $|\Gamma|>|E|$.

However the proof of this theorem is less elementary; in particular, some form of the axiom of choice must be used, since the theorem implies that every set can be ordered.

A direct proof for (*) may be given as follows: if $\phi: P(E) \rightarrow E$ satisfied (4), then the sequence of subsets defined for all ordinals $\alpha$ by the transfinite recursion

$$
X_{\alpha}=\left\{\phi\left(X_{\xi}\right): \xi<\alpha\right\}
$$

would be strictly increasing, which is impossible.

3. Solution of a problem of Lawvere. If $E$ and $F$ are partially ordered sets then the cardinal power $F^{E}$ is the set of all homomorphisms from $E$ to $F$, with the partial order defined by setting $f \leqq g$ whenever $f(x) \leqq g(x)$ for all $x \in E$.

The $n$-element chain will be denoted by $n$. It is clear that $2^{E}$ is isomorphic to $R(E)$. A partially ordered set $E$ is said to be discretely ordered if, for all $x, y \in E$

$$
x \leqq y \text { iff } x=y .
$$

THEOREM 3. If $E$ and $F$ are partially ordered sets and $F$ is not discretely ordered then there is no weak monomorphism from $F^{E}$ to $E$. In particular, there is no injective homomorphism from $F^{E}$ to $E$ and no surjective homomorphism from $E$ to $F^{E}$.

Proof. Suppose there exists a weak monomorphism $\phi: F^{E} \rightarrow E$. Since $F$ is not discretely ordered it contains a subset isomorphic to 2 . Hence $F^{E}$ contains a subset isomorphic to $2^{E}$, or what is the same, to $R(E)$. By restricting $\phi$ we obtain a weak monomorphism from $R(E)$ to $E$, contrary to Theorem 2 .

We now consider the case where $F$ is discretely ordered. Let $D$ be the set of connected components of $E$ (that is, the equivalence classes under the equivalence relation generated by $\leqq$ ), with the discrete partial order. It is clear that $F^{E}$ is isomorphic to the discretely ordered set $F^{D}$. Any surjective homomorphism from $E$ to $F^{D}$ induces a surjective homomorphism from $D$ to $F^{D}$; hence bv Cantor's Theorem there exists a surjective homomorphism from $E$ to $F^{E}$ just when $F=1$. There exists an injective homomorphism from $F^{D}$ to $E$ if and only if $\left|F^{D}\right| \leqq|E|$; hence there exists an injective homomorphism from $F^{E}$ to $E$ just when $\left|F^{D}\right| \leqq|E|$. 
As a result of the above discussion we have the following proposition:

If $E$ and $F$ are partially ordered sets, then there is no surjective homomorphism $\phi: E \rightarrow F^{E}$ unless $F=1$.

As mentioned in the introduction this answers a question posed by Lawvere. We observe that the result can be derived directly from $(\rho)$ without the use of the more general Theorem 2; for if $F$ is not discretely ordered then there exists a surjective homomorphism from $F^{E}$ to $2^{E}$.

\section{REFERENCES}

1. A. C. Davis, A characterization of complete lattices, Pacific J. Math. 5 (1955), 311-319. MR 17, 574.

2. A. M. Gleason and R. P. Dilworth, $A$ generalized Cantor theorem, Proc. Amer. Math. Soc. 13 (1962), 704-705. MR 26 \#2365.

3. W. Sierpinski, Sur un probleme concernant les sous-ensembles croissants $d u$ continu, Fund. Math. 3 (1922), 109-112.

4. A. Tarski, A lattice-theoretical fixpoint theorem and its applications, Pacific J. Math. 5 (1955), 285-309. MR 17, 574.

UNIVERSITY OF LEICESTER, LEICESTER, ENGLAND 